\title{
Cirurgia de revascularização miocárdica: mudanças na vida de indivíduos e o cuidado de si
}

\author{
Myocardial revascularization surgery: individual life changes and self-care \\ Cirugía de revascularización miocárdica: cambios en la vida de individuos y el cuidado de sí
}

\author{
Claudia Regina Maldaner'; Margrid Beuter"; Nara Marilene Oliveira Girardon-Perlini"l'; \\ Eliane Raquel Rieth Benetti'v; Macilene Regina Paulettov; Matheus Souza Silva ${ }^{\text {vI }}$
}

\begin{abstract}
RESUMO
Objetivo: analisar as mudanças provocadas pela Cirurgia de Revascularização Miocárdica (CRM) e o cuidado de si de indivíduos revascularizados. Método: pesquisa qualitativa realizada com dez indivíduos pós CRM, em um hospital escola do Sul do Brasil. Os dados foram coletados por meio de entrevista narrativa e submetidos à análise temática. Projeto aprovado por Comitê de Ética e Pesquisa Resultados: da análise emergiu a categoria, mudanças provocadas pela CRM e o cuidado de si de indivíduos revascularizados com as subcategorias: agora eu me cuido; a mão dupla do cuidado; a imposição do cuidado; a valorização da vida; mudança de atitude nos relacionamentos; e, a família mais próxima. Conclusão: após a cirurgia, os indivíduos passam a repensar suas atitudes consigo e com as outras pessoas, valorizando sua vida, a saúde e a família. Os bens materiais e problemas que permeiam o cotidiano tornam-se menos relevantes, procurando assumir atitudes que lhe tragam prazer e bem-estar. Descritores: Enfermagem; revascularização miocárdica; cuidados de enfermagem; cardiologia.
\end{abstract}

\section{ABSTRACT}

Objective: to examine changes brought about by myocardial revascularization surgery (coronary artery bypass graft, CABG) and self-care by revascularized individuals. Method: in this qualitative study of ten individuals post-CABG at a teaching hospital in southern Brazil, data were collected by narrative interview and submitted to thematic analysis. Results: analysis revealed the category "changes caused by CABG, and revascularized individuals self-care" with the subcategories "now I take care of myself"; "care as two-way process"; "care as imposition"; "valuing life"; "changed attitude in relationships"; and "closest relatives". Conclusion: after surgery, individuals begin to rethink their attitudes to themselves and other people, valuing their life, health and family. Material possessions and everyday problems become less relevant, and individuals endeavor to act in ways that bring pleasure and well-being.

Descriptors: Nursing; myocardial revascularization; nursing care; cardiology.

\section{RESUMEN}

Objetivo: analizar los cambios producidos por la cirugía de revascularización miocárdica (CRM) y el cuidado de sí de los individuos revascularizados. Método: investigación cualitativa realizada con diez individuos después de CRM, en un hospital universitario en el sur de Brasil. Los datos fueron recolectados por medio de una entrevista narrativa y sometidos al análisis temático. Resultados: del análisis surgió la categoría cambios causados por el CRM y el cuidado de sí de los pacientes revascularizados con subcategorías: ahora me cuido; la doble vía del cuidado; la imposición del cuidado; la valorización de la vida; la actitud en los relacionamientos y la familia más cerca. Conclusión: Después de la cirugía, las personas comienzan a replantearse sus actitudes con usted y otras personas, valorando su vida, la salud y la familia. Materiales y mercancías temas que permean la vida cotidiana se vuelve menos relevante, buscando tomar acciones que dan placer y bienestar. Descriptores: Enfermería; revascularización miocárdica; atención de enfermeira; cardiología.

\section{INTRODUÇÃO}

A Cirurgia de Revascularização Miocárdica (CRM) têm modificado a sobrevida dos pacientes com expressiva redução da morbimortalidade após eventos cardiovasculares $^{1}$. Essa cirurgia objetiva reestabelecer a perfusão das artérias coronárias e aliviar sintomas anginosos, com melhora da qualidade de vida do indivíduo revascularizado².

Tanto os eventos cardiovasculares quanto a cirurgia cardíaca interferem no modo de viver do indivíduo, pois é necessário reestruturar o estilo de vida, o que de-

'Enfermeira. Mestre em Enfermagem. Universidade Federal de Santa Maria, Hospital Universitário de Santa Maria. Rio Grande do Sul, Brasil. E-mail: claumaldaner@yahoo.com.br

"Enfermeira. Doutora em Enfermagem. Professor Associado da Universidade Federal de Santa Maria. Rio Grande do Sul, Brasil. E-mail: margridbeuter@gmail.com I'Enfermeira. Doutora em Enfermagem. Professor Adjunto da Universidade Federal de Santa Maria. Rio Grande do Sul, Brasil. E-mail: nara.girardon@gmail.com IVEnfermeira. Doutoranda em Enfermagem. Universidade Federal de Santa Maria, Hospital Universitário. Rio Grande do Sul, Brasil. E-mail: elianeraquelr@yahoo.com.br ${ }^{\vee}$ Enfermeira. Mestre em Enfermagem. Universidade Federal de Santa Maria, Hospital Universitário de Santa Maria. Rio Grande do Sul, Brasil. E-mail: macipauletto@gmail.com v'Enfermeiro. Hospital Universitário de Rio Grande. Rio Grande do Sul, Brasil. E-mail: matheus_souza_silva@hotmail.com 
manda estratégias de enfrentamento ${ }^{3-4}$. Entre as modificações, cita-se a necessidade de mudança de hábitos, afastamento das atividades laborais, cuidados com a saúde e uso contínuo de medicações. Nesse sentido, a cirurgia cardíaca determina novas percepções sobre a vida e modo de viver. Essa adaptação depende de características individuais, redes de apoio e comorbidades associadas. Dessa forma, a experiência cirúrgica poderá ser transformadora para alguns, ter pouca influência para outros, sendo que o modo de enfrentamento de cada um determina o desempenho do cuidado de si.

Diante das demandas de cuidado no pós-operatório, é importante que os profissionais de saúde, especialmente os enfermeiros, conheçam as alterações que ocorrem na vida desses indivíduos e as implicações para o cuidado de si. O enfermeiro pode auxiliar os indivíduos no enfrentamento da patologia e no processo cirúrgico de CRM, porque é ele quem planeja e organiza a assistência e assegura que a equipe de enfermagem faça uma abordagem individual e integral ${ }^{5}$. Como a enfermagem representa um dos elos entre o paciente, ambiente e familiares, pontua-se que é possível qualificar o cuidado, tanto assistencial quanto de educação para a saúde, instrumentalizando os indivíduos pós CRM para o cuidado de si.

Diante desses aspectos, objetivou-se analisar as mudanças provocadas pela CRM e o cuidado de si de indivíduos revascularizados.

\section{RefERENCIAL TEÓRICO}

Para Foucault, o cuidado de si é definido como uma atitude para consigo, para com os outros e para com o mundo e, designa o conjunto de condições de espiritualidade, transformações de si que constituem uma condição para se ter acesso à verdade ${ }^{6}$. 0 autor salienta que a regra geral do cuidado de si é ocupar-se de si mesmo, não esquecer-se de si e ter cuidado consigo.

Neste estudo, o cuidado de si está configurado na perspectiva da autonomia dos sujeitos revascularizados. A autonomia, compreendida como algo inerente ao ser humano, ou passível de ser desenvolvido ao longo da vida e importante para superação das dificuldades diárias no processo de recuperação. O cuidado de si é percebido como uma atitude desenvolvida em todas as situações de vida dos sujeitos, de acordo com suas condições de vida naquele momento. Nesse sentido, entende-se que a enfermagem pode fazer-se presente, utilizando a educação em saúde para instrumentalizar os indivíduos que realizaram CRM, no desempenho do cuidado de si.

\section{MetOdologia}

Pesquisa qualitativa, descritiva, realizada com 10 indivíduos pós CRM assistidos em hospital escola do Rio Grande do Sul. Foram incluídos indivíduos que realizar- am CRM, com no máximo 120 dias de pós-operatório, com consulta médica agendada no ambulatório de cirurgia cardíaca no período de coleta de dados e que se apresentavam lúcidos, orientados, em condições de expressar-se verbalmente.

Para seleção dos participantes, acessou-se a agenda de consultas e verificou-se no prontuário aqueles que atendiam os critérios de inclusão, sendo estes convidados a participar da pesquisa por meio de contato telefônico. Os dados foram obtidos por meio da entrevista narrativa, que se caracteriza pela lembrança das pessoas do que aconteceu, que sequenciam suas experiências e são capazes de incorporar significado e intencionalidade aos seus atos, relações e estruturas sociais ${ }^{7}$.

Os participantes foram esclarecidos quanto a pesquisa e assinaram o Termo de Consentimento Livre e Esclarecido. As entrevistas foram realizadas em sala reservada, em horário conveniente para o participante, gravadas e transcritas integralmente. As entrevistas foram codificadas com a letra "E" de entrevistado e o número sequencial da ocorrência das mesmas (Ex: E1, E2, E3), para assegurar anonimato.

O conteúdo das entrevistas foi analisado por meio da análise temática ${ }^{8}$, caracterizada por dois níveis de interpretação: as determinações fundamentais da pesquisa, que observa o contexto sociohistórico do grupo social em questão e o interpretativo, que representa o encontro com os fatos empíricos e procura no relato dos informantes o sentido, a lógica interna, as projeções e as interpretações ${ }^{8}$. A fase interpretativa dividiu-se em ordenação e classificação dos dados ${ }^{7}$, dando origem a uma categoria com seis subcategorias, analisadas na próxima seção.

Os princípios éticos da Resolução no 466/2012 foram respeitados e o estudo foi aprovado por Comitê de Ética sob no 10993912.6.0000.5346.

\section{RESUltados E Discussão}

Participaram do estudo sete homens e três mulheres, com idade entre 45 e 58 anos. Em relação ao estado civil, seis eram casados, dois divorciados, um solteiro e um viúvo. Seis dos participantes tinham filhos. Quanto à procedência, seis residiam no município da coleta de dados. Todos os participantes estavam afastados de suas atividades laborais, sendo que quatro eram aposentados, três recebiam auxílio doença, dois realizavam atividades do lar e um intitulou-se desocupado.

A partir da análise das entrevistas emergiu a categoria temática, mudanças provocadas pela CRM e o cuidado de si de indivíduos revascularizados com as subcategorias: agora eu me cuido; a mão dupla do cuidado; a imposição do cuidado; a valorização da vida; mudança de atitude nos relacionamentos; e, a família mais próxima. 


\section{Agora eu me cuido}

Após ocorrência de evento cardiovascular e necessidade da CRM, o cuidado de si torna-se prioridade para os participantes, uma vez que passaram por situações ameaçadoras da vida.

Esse susto que tive, eu podia ter morrido! A morte é apavorante! Estou aqui conversando contigo e há um mês atrás estava praticamente estava morta. Agora estou me cuidando, somente de mim, da minha saúde. (E4)

Tu não estás dando muita bola para ti, mais para os outros. Depois que tu vê aquilo que tu passou, tu vê que tem que dar mais valor para a gente. (E5)

Constata-se nas falas descritas, que o susto, como se referem a CRM, fez com que percebessem a necessidade de cuidarem de si. Antes desse evento, não se colocavam como o centro de suas vidas, pois estavam mais preocupados com os outros do que consigo. Nesse sentido, Foucault afirma que "é preciso que te ocupes contigo mesmo, que não te esqueças de ti mesmo, que tenhas cuidado consigo mesmo"6:6. O cuidado de si implica em preocupar-se consigo, ser agente ativo e efetivo do próprio cuidado.

Também é manifestado o desejo de recuperar e manter a saúde, com propósito de mudar atitudes, empregando o cuidado de si como estratégia.

Eu tenho que me cuidar, senão eu morro! Eu peço para Deus para não chegar essa hora. Eu gostaria de viver um pouco mais aqui, vou me cuidar para viver um pouco mais. Agora já penso mais, vou tocar a minha vida e me cuidar mais. (E7)

Resultados semelhantes apontam que vivenciar uma situação, como a cirurgia cardíaca, traz mudanças significativas para a vida dos pacientes que vão desde transformações físicas até biopsicossociais, que requerem a instituição de novos hábitos de vida ${ }^{9}$. Nessa conjuntura, os profissionais de saúde têm papel essencial na orientação e conscientização dos revasculariza-dos sobre aspectos relacionadas à alimentação, prática de exercícios físicos e hábitos de vida, a fim de auxiliá-los nas mudanças necessárias para uma vida saudável ${ }^{10}$. Além disso, os enfermeiros podem auxiliar os pacientes, propondo espaços de discussões para trocar ideias, compartilhar vivências, minimizar angústias e instrumentalizá-los para o cuidado de si e impulsionálos a repensar e a adquirir novas práticas.

\section{A mão dupla do cuidado}

Os participantes reconhecem e valorizam o cuidado recebido dos profissionais e outras pessoas, porém entendem que a atitude principal depende deles.

É só obedecer às regras, porque o bem é para mim. Médicos e enfermeiras ajudam a gente, mas temos que nos ajudar também. É como dizem: Deus te ajuda, mas tens que se ajudar também. (E1)

No pós-operatório de CRM os indivíduos percebem-se como protagonistas do cuidado e é indispen- sável que estejam preparados para isso. Os enfermeiros têm papel fundamental nas orientações, no incentivo à autonomia e estabelecimento de estratégias de cuidado. Preconiza-se uma abordagem individualizada, na qual o enfermeiro empregue conhecimentos e competências para melhorar a qualidade da assistência e obter resultados satisfatórios no cuidado de si dos indivíduos ${ }^{11}$.

Os participantes do estudo compreendem que o cuidado não pode ser unilateral e afirmaram que, além das ações das outras pessoas, a decisão e o compromisso de cuidar de si tem que partir deles.

Passei por tudo isso, passei trabalho, o médico se esforçou para me ajudar. Deus está me ajudando! E agora? Eu não faço a minha parte? Eu vou ter que fazer! Tanta gente está aqui! Tu estás me ajudando! Eu tenho que entender isso. Eu quero fazer tudo diferente agora. (E2)

Porque se eu não me cuidar, não adianta os outros quererem me cuidar. (E8)

Os profissionais de saúde são percebidos como alguém que confia na capacidade dos pacientes cuidarem de si e, por isso, investem orientações e expectativas. Destaca-se que quando os enfermeiros demonstram empatia nas ações, favorecem a verbalização de preocupações, a construção de um cuidado embasado nas necessidades individuais e expande a adesão ao tratamento. Dessa maneira, as ações de ensino, orientação e educação que visam a manutenção do processo de vida e funcionamento do ser humano incentivam o protagonismo do cuidado ${ }^{10,12}$.

Ademais, quando as orientações são específicas e fundamentadas nas características de determinada população, reduz as barreiras da orientação empírica e despersonalizada, fortalece o foco em estratégias interessantes para paciente-família e aumenta a segurança, praticidade e confiança na execução do cuidado ${ }^{12}$.

\section{A imposição do cuidado}

Os participantes relataram que, por momentos, o cuidado dos familiares foi percebido como excessivo e impositivo e, que algumas atividades poderiam ser realizadas sem auxílio.

Eu estou sendo muito paparicado. A mulher ficou me cuidando demais. Qualquer coisinha que eu precisar, um gole de água, não deixa que eu pegue. Ela deixa de fazer as coisas dela para ficar em volta de mim. (E3)

Estão me cuidando que nem criança. Não deixam descascar uma laranja, pegar uma faca. Para levantar, alguém pega pela mão; ir no banheiro, outro leva. Parece que agora eu não sou a mãe, sou filha. (E5)

Após a CRM, os participantes sentem-se superprotegidos e referem uma inversão de papéis. Salientam que os familiares na tentativa de ajudar, por momentos, exageram nos cuidados, o que implica na diminuição da autonomia do indivíduo. Nesse sentido, sabe-se que com o adoecimento emerge um processo coletivo de sofrimento em que família e indivíduo padecem para 
lidar com o problema e compartilham bilateralmente medos, incertezas e preocupações ${ }^{13}$.

Além disso, o componente familiar assume importância como apoio, desde o diagnóstico até o enfrentamento das mudanças do novo contexto de vida ${ }^{14}$. Assim, é importante que os profissionais compreendam como o indivíduo no pós CRM lida com o processo cirúrgico e com o retorno ao ambiente familiar, a fim de favorecer a abordagem dos envolvidos, estimulando o cuidado, mas principalmente a autonomia e independência dos indivíduos.

Alguns indivíduos revascularizados mantiveram uma postura de obediência aos familiares, enquanto outros ficaram descontentes e mobilizaram-se para manter a autonomia para tarefas que se sentiam aptos.

Em casa está todo mundo com muito cuidado comigo, até para eu levantar. Quando falo em tomar uma água, quando eu vejo, o copo já está ali. Só não me dão o banho porque eu digo: 'Não! Vou tomar meu banho sozinha!'. (E4)

Essa manifestação corrobora com resultados de estudo ao afirmar que, apesar do cuidado e da cooperação familiar favorecer a recuperação do paciente, situações de atrito podem causar prejuízos emocionais, de interação social e familiar, em especial pelas cobranças sobre mudanças no estilo de vida ${ }^{15}$. Por isso, é fundamental que, os indivíduos adquiram autonomia e recomecem, aos poucos, a desempenhar tarefas de vida cotidiana, procurando uma continuidade, embora algumas trajetórias necessitam sem pensadas e ressignificadas ${ }^{4}$. Diante disso, é necessário que os profissionais de saúde instrumentalizem os familiares acerca da importância da autonomia do indivíduo revascularizado, oportunizando o cuidado de si e o cuidado compartilhado.

\section{A valorização da vida}

A realização da CRM sinaliza que a vida é finita. Essa percepção implica em maior valorização da vida após a cirurgia.

Como diz o meu marido: 'Já estamos velhos, 40 anos de casados, vamos passear, sair, aproveitar a vida, porque a vida é curta.' A gente dá valor para a vida, quando enfrenta uma situação dessas. (E5)

A gente dá mais valor à vida. Agora que eu me sinto renovado, porque eu vou continuar com o problema [cigarro]? Se eu sei que pode ocasionar novamente. Sejamos um pouquinho conscientes e inteligentes. Larguemos desses vícios. (E6)

Os participantes revelaram a intenção para modificar hábitos de vida, abandonar vícios e superar fatores estressantes do dia a dia. Essa disposição identificada vem ao encontro de pesquisa que evidenciou o desejo manifestado por pacientes de aproveitar as coisas boas que a vida pode oferecer e realizar outras que ainda não haviam se dado o tempo de fazer pós $\mathrm{CRM}^{9}$.
Ao perceber que a continuidade da vida pode estar ameaçada, e que para preservá-la necessita mudar hábitos, um participante demonstra desejo de cuidar de si, rever alguns comportamentos que podem ser prejudiciais e aproveitar as coisas boas da vida.

Sei que pode acontecer algum imprevisto. Eu tenho aquela consciência que eu não sou mais 'super-homem' como achava que era, que não era qualquer bobagem que ia me levar. Foi as bobagens que quase me levaram, fui eu mesmo que fiz, com alimentação desregrada, não me cuidando. Aquela ansiedade, angústia, mudou total. Tem uma música que diz: 'Ando devagar porque já tive pressa...' (E10)

Incluem-se nessas ações, além da valorização da vida o desejo manifestado de estar perto da família, a mudança de estilo de vida, como alteração quanto ao hábito de fumar, o controle do estresse, a dieta, a prática de exercícios físicos e uso correto de medicamentos. Esse resultado corrobora que além de visualizar mudanças, os indivíduos buscam também ressignificar suas vidas4. Esses dados apontam que ocorre uma revisão de valores e de postura em relação aos bens materiais, os quais tornaram-se secundários após a CRM.

Às vezes ela [esposa] não pode fazer, tem serviços que ela não faz. Ela largou tudo, praticamente. Mas é melhor estar vivo, do que ter dinheiro a mais no bolso. (E1)

Eu não penso em fazer capital, fazer dinheiro, comprar terra e coisas assim. Isso eu não quero mais. Até tinha planos, tenho as terras, tem as terras dos meus irmãos, eu tinha vontade de comprar uma parte deles, mas hoje eu não quero mais. (E9)

Parece que o cuidado de si se torna prioridade quando a reflexão sobre o sentido da vida e os valores que devem ser preservados se sobrepõe ao valor atribuído aos bens materiais. Foucault salienta que "é preciso que não te esqueças de ti mesmo"6:7. Nesse aspecto, os profissionais de saúde podem reforçar a motivação manifestada pelos indivíduos de se cuidar e aproveitar as coisas boas da vida. Isso, certamente, implicará em uma melhor qualidade de vida e adesão ao tratamento.

\section{Mudança de atitude nos relacionamentos}

Os indivíduos pós CRM mudaram a convivência com as pessoas e com problemas do dia a dia, procuraram não se estressar com pequenas coisas e perceberam-se mais amorosos.

Eu achava coisa onde não existia. Não tinha calma, paciência e agora não! Não esquento mais a cabeça como esquentava com coisas que não existem. Colocava aquilo na cabeça e tinha que ser assim. Agora não, eu encosto a cabeça no travesseiro e durmo bem tranquilo. (E1)

Perdi muita amizade, por eles fazerem assim e eu corrigir. Eu tinha uma mania assim, um irmão meu não pode errar! Sabe, sobre isso eu aprendi muito. Eu fiquei mais amoroso. A minha mulher, agora eu valorizo o dobro e as pessoas em volta também. Eu estou vivendo uma vida nova. (E2) 
A experiência da CRM ajudou os indivíduos a entender melhor e aceitar as outras pessoas e a si mesmas, resultado que coadune com estudo que identificou as expectativas de pacientes revascularizados no momento da alta hospitalar ${ }^{4}$. Isso também faz parte do cuidado de si, a busca por tornar-se melhor como ser humano e como cidadão. Esta nova concepção da vida aponta que os indivíduos perceberam a melhora no relacionamento com a família pós CRM, e demonstraram o desejo de aproveitar o dia com eles. Ademais, estão mais abertos, no sentido de buscar a recuperação de laços de afeto, amor, apoio e solidariedade.

Hoje eu sinto mais essa coisa boa de ajudar, porque fui ajudado. Como é bom quando a gente está sendo auxiliado. Depois da cirurgia senti que mudou muita coisa na minha vida, positivamente. Hoje entendo mais o ser humano. (E6)

Me fez melhorar o trato com as pessoas. Interajo mais com os meus filhos. Eu atribuo isso à cirurgia e o que vem na volta. (E10)

Pontua-se que a harmonia nos relacionamentos não é regra, pois em oposição a esse resultado, estudos constataram o afastamento do indivíduo revascularizado do convívio familiar, dos filhos, netos, amigos, das reuniões sociais e confraternizações ${ }^{16}$. $\mathrm{O}$ adoecimento pode interferir nas práticas de lazer e integração social, dificultar as interações entre integrantes da família e destes para com a rede social ${ }^{17}$. Os profissionais devem atentar para essas questões, estimular o exercício do cuidado de si desses indivíduos, para que se sintam importantes ao conviver com sua rede social, buscando atividades de lazer que proporcionem satisfação e prazer.

\section{A família mais próxima}

O cuidado recebido dos familiares foi percebido como positivo, associado ao amor, carinho, companheirismo e proximidade.

Então, eu só posso dizer que estou feliz da vida, com meus filhos, meus netos, todos em volta de mim. É numa hora dessas que tu vê o que é o amor e o carinho de um filho, de um neto. Aquilo ali ajuda muito. (E5)

Após a CRM, os participantes perceberam seus familiares mais cuidadosos e carinhosos com eles. Resultado convergente aponta que a família é vista como a fonte de apoio mais importante ${ }^{18}$. Nesse contexto, salientam que é nos momentos difíceis que descobrem quem realmente é importante na vida.

Minha esposa é uma pessoa maravilhosa, sempre me assistiu, principalmente agora. Então eu vi, realmente, a pessoa que eu tenho junto comigo, porque é no momento que a gente mais precisa que a gente vê as pessoas que tu tem em casa. (E6)

O companheirismo também da minha esposa comigo, para todas as horas, os meus filhos. Eu andava meio afastado e agora não, tudo por conta da cirurgia. (E10)
O evento cardíaco tem impacto sobre as relações conjugais e o estresse causado pela doença pode fortalecer os laços entre os cônjuges e familiares. Verificou-se que as famílias fortificaram seus vínculos após a CRM e os familiares empenharam-se para estarem próximos. Aponta-se que as relações familiares interferem positivamente na qualidade de vida dos pacientes submetidos à CRM, principalmente no aspecto psicológico ${ }^{19}$. Isso revela que o enfermeiro, além de estabelecer estratégias para incentivar o cuidado de si, pode auxiliar paciente e familiares a fortalecerem os vínculos.

Destaca-se que a equipe de enfermagem possui conhecimento, habilidade e capacidade de comunicação e compreensão para fornecer essas orientações e, que o enfermeiro é um elo fundamental na busca de ações que visem uma assistência integral ${ }^{20}$. Para isso é importante conhecer as mudanças enfrentadas pelos indivíduos revascularizados, compreender as ações e reações humanas, considerando os fatores que podem interferir no cuidado de si.

\section{CONCLUSÃO}

Após a CRM ocorreram mudanças na vida dos indivíduos que os fizeram repensar suas atitudes consigo e com outras pessoas. A percepção da finitude possibilitou um olhar diferenciado sobre sua vida, que desencadeou o desejo de cuidar de si para manutenção da saúde. Os indivíduos revascularizados perceberam-se como protagonistas do cuidado, respaldados pela parceria com os profissionais de saúde, mas apontaram a imposição de cuidados a que algumas vezes são submetidos pelos familiares, o que lhes tolhe a liberdade de escolha e o próprio cuidado consigo.

Os resultados indicam maior valorização da vida pós CRM, quando comparada aos problemas do cotidiano e bens materiais. Além disso, percebe-se melhora nos relacionamentos e o desejo de viver mais próximo das pessoas com as quais tem afinidade, amizade e afeto. $O$ cuidado de si se manifesta quando entendem que precisam adotar uma postura ativa e autônoma em todas as situações da sua vida, tomando posse de si e de seu cuidado, assumindo atitudes que lhe tragam prazer e bem-estar.

Diante destes resultados, destaca-se a importância de os profissionais de saúde conhecerem as mudanças na vida de indivíduos revascularizados, para que possam auxiliá-los a superarem suas dúvidas, conflitos e medos. O enfermeiro pode promover grupos de discussão e também, nas consultas de enfermagem, incentivar para que essas mudanças de atitude, não esmoreçam com o passar dos dias e o cuidado de si seja exercido com confiança, responsabilidade e autonomia.

Este estudo apresenta como limitações o quantitativo de indivíduos revascularizados e o intervalo de tempo desde sua realização, entretanto considera-se esses achados relevantes para o cuidado de enfermagem pós CRM e para adensar o conhecimento de enfermagem nessa área. 


\section{REFERÊNCIAS}

1. Braile DM, Gomes WJ. Evolution of cardiovascular surgery. The Brazilian saga. A History of work, pioneering experience and success. Arq. Bras. Cardiol. 2010;94(2):151-2.

2. Dallan LAO, Jatene FB. Revascularização miocárdica no século XXI. Rev. Bras. Cir. Cardiovasc. 2013;28(1):137-44.

3. Koerich C, Baggio MA, Erdmann AL, Lanzoni GMM, Higashi GDC. Revascularização miocárdica: estratégias para o enfrentamento da doença e do processo cirúrgico. Acta Paul. Enferm. 2013;26(1): 8-13.

4. Cielo C, Silveira M, Arboit EL, Camponogara S. Expectations of patients submitted to myocardial revascularization surgery at the time of hospital discharge. Rev. pesqui. cuid. fundam. (Online). 2015 (citado em 06 mai 2016). 7(3):2670-87. Disponível em: http://www.seer.unirio.br/index.php/cuidadofundamental/ article/view/3623

5. Barretta JC, Auda JM, Barancelli MDC, Antoniolli D. Postoperative in cardiac surgery: reflecting about nursing care. Rev. pesqui. cuid. fundam. (Online) 2017 (citado em 20 ago 2018); 9(1):259-64. Disponível em: http://seer.unirio.br/index.php/ cuidadofundamental/article/view/4042

6. Foucault M. A hermenêutica do sujeito. 3a ed. São Paulo : Martins Fontes; 2010.

7. Bauer MW, Gaskell G. Pesquisa qualitativa com texto, imagem e som: um manual prático. 10a ed. Petrópolis (RJ): Vozes; 2012.

8. Minayo MCS. O desafio do conhecimento: pesquisa qualitativa em saúde. 14a ed. São Paulo: Hucitec; 2014.

9. Moura RS, Saraiva FJC, Santos RM, Rosa RFN, Rocha KRSL, Cavalcanti RC. Experiences of patients after myocardial revascularization surgeries: life stories. Rev. enferm. UFPE on line (Online) 2017 (citado em 20 ago 2018); 11(Supl. 12):5221-30. Disponível em: https://periodicos.ufpe.br/revistas/revistaenfermagem/ article/view/22863

10. Baggio MA, Erdmann AL, Higashi GDC, Koerich C, Lanzoni GMM. Discovery of cardiovascular disease: associating causes and experiencing the context of a referral hospital. Rev. enferm. UERJ. 2016; 24(4):e7952.

11. Lima FET, Araújo TL, Lopes MVO, Silva LF, Monteiro ARM, Oliveira SKP. Risk factors of coronary disease in patients who have undergone myocardial revascularization. Rev Rene. 2012; 13(4):853-60.
12. Gentil LLS, Silva RM, Benavente SBT, Costa ALS. Educational manual for the care of patients in the post-operative period of myocardial revascularization: a tool for patients and families. Rev. eletrônica enferm. (Online) 2017 (citado em 20 ago 2018). 19:a38. Disponível em: https://revistas.ufg.br/fen/article/download/43068/24659

13. Montoro CH, González JS, Amezcua M, Nieves CB, Montero SP, Mañas MC. Understanding the suffering of a patient with an illness: signs, context and strategies. Rev. latinoam. enferm. (Online) 2012 (cited in 2016 mai 21) 20(3):619-28. Available from: http://www.scielo.br/pdf/rlae/v20n3/a26v20n3.pdf

14. Cunha KS, Erdmann AL, Higashi GDC, Baggio MA, Kahl C, Koerich $C$ et al. Revascularização do miocárdio: desvelando estratégias de referência e contrarreferência na atenção primária à saúde. Rev. baiana enferm. 2016; 30(1):295-304.

15. Erdmann AL, Lanzoni GMM, Callegaro GD, Baggio MA, Koerich C. Compreendendo o processo de viver significado por pacientes submetidos a cirurgia de revascularização do miocárdio. Rev. latinoam. enferm. (Online) 2013 (citado em 21 mai 2016). 21(1):1-8. Disponível em: http://www.scielo.br/pdf/rlae/v21n1/ pt_v21n1a07

16. Callegaro GD, Koerich C, Lanzoi GMM, Baggio MA, Erdmann AL. Significando o processo de viver a cirurgia de revascularização miocárdica: mudanças no estilo de vida. Rev. gaúch. enferm. 2012; 33(4):149-56.

17. Garcia RP, Budó MLD, Simon BS, Wünsch S, Oliveira SG, Barbosa MS. Vivências da família após infarto agudo do miocárdio. Rev. gaúch. enferm. 2013; 34(3):171-78.

18. Bin G, Costa MCS, Vila VSC, Dantas RAS, Rossi LA. Meanings of social support for people submitted to coronary artery by-pass graft surgery: ethnographic study. Rev. bras. enferm. (Online) 2014 (cited in 2016 maio 21). 67(1): 71-7. Available from: http://www.scielo.br/pdf/reben/v67n1/0034-7167-reben-67-01-0071.pdf

19. Alves MO, Lanzoni GMM, Koerich C, Higashi GDC, Baggio MA, Erdmann AL. The process of experiencing the coronary revascularization surgery: an analysis of gender. Esc. Anna Nery Rev. Enferm. 2016;20(4):e20160093.

20. Lira TDA, Negro-Dellacqua M, Santos VEFA. Cardiac catheterization: experience faced by hospitalized patients. Rev. enferm. UERJ, Rio de Janeiro, 2017; 25:e15608. 